\title{
Iris Recognition using Convolutional Neural Network
}

\author{
Md. Shafiul Azam \\ Computer Science and Engineering, \\ Pabna University of Science and Technology, \\ Bangladesh
}

\author{
Humayan Kabir Rana \\ Computer Science and Engineering, \\ Green University of Bangladesh, Bangladesh
}

\begin{abstract}
It is indispensable to ensure security biometrically in the most authentication and identification scenario. Iris recognition regarded as the most reliable biometric recognition due to its stable and extraordinary variation in texture. The unique patterns are used in iris recognition to identify individuals in requiring a high level of security. This paper explores an efficient technique that uses convolutional neural network $(\mathrm{CNN})$ and support vector machine (SVM) for feature extraction and classification respectively to increase the efficiency of recognition. The proposed technique has been successfully applied and also clearly demonstrates the performance of the experimental evaluation on iris images from the CASIA database.
\end{abstract}

\section{Keywords}

Iris Recognition, Hough Transformation, Daugman's Rubber Sheet Model, CNN and SVM

\section{INTRODUCTION}

Biometric technology is concerned with recognizing the identity of individuals based on their unique physical or behavioral characteristics [1]. The physical characteristics such as iris, face, fingerprint, retina, vein and hand geometry or the behavioral characteristics such as hand writing, human gait, signature, and keystrokes have unique, accurate and stable information about a person to be used in authentication applications. The growing developments in information technology have made it possible to use biometrics in applications where it is required to establish or confirm the identity of individuals [2]. Now a day, the increasing demand for enhanced security in the daily life towards the digitalization has directed the improvement of the reliable and intelligent person identification system based on biometrics. The cards or passwords are used in traditional identification systems. This traditional system may be broken down by losing or stealing cards and failing to remember password. That is why biometric identification systems that can identify humans without depending on what person possesses or what person remembers is badly needed.

Applications such as passenger control in airports, access control in restricted areas, border control, database access and financial services are some of the examples where the biometric technology has been applied for more reliable identification and verification [2]. The biometric technology, in the field of financial services, has shown a great potential in offering more comfort to customers while increasing their security. For an example, banking services and payments based on biometrics are going to be much safer, faster and easier than the existing methods based on credit and debit cards. Although there are still some concerns about using biometrics in the mass consumer applications due to information protection issues, it is believed that the technology will find its way to be widely used in so many different applications [3]. The biometric technologies provide more secure than the use of passwords and comfortable accessibility and have dealt with problems such as forgetting or hacking passwords [3].

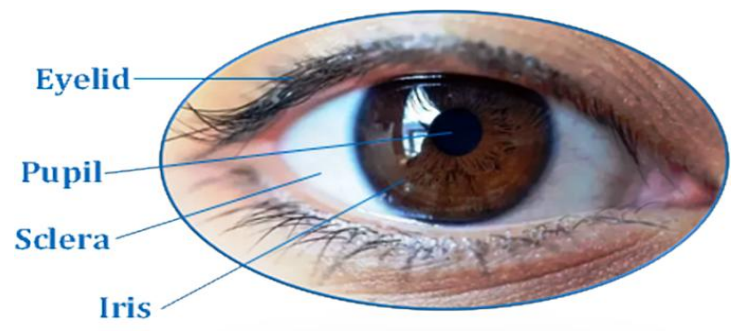

Fig 1: The outer structure of human iris.

The iris pattern belong the most enviable properties for verification comparing other biometrics because of its uniqueness, stability over time and relatively easy accessibility as shown in Table 1. It is seen that it has the highest accuracy among all biometrics. This fact motivates us to select iris recognition as a research study.

Table 1: Comparison of properties of several biometric identifiers [4].

\begin{tabular}{|c|c|c|c|c|c|c|c|c|}
\hline $\begin{array}{l}\text { Biomet } \\
\text { ric } \\
\text { identifi } \\
\text { er }\end{array}$ & 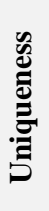 & 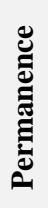 & 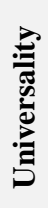 & 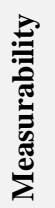 & 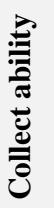 & 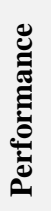 & 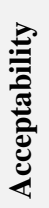 & 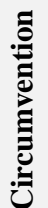 \\
\hline Iris & $\mathrm{H}$ & $\mathrm{H}$ & $\mathrm{H}$ & $\mathrm{M}$ & $\mathrm{H}$ & $\mathrm{H}$ & $\mathrm{M}$ & $\mathrm{L}$ \\
\hline Retinal & $\mathrm{H}$ & $\mathrm{H}$ & $\mathrm{H}$ & $\mathrm{L}$ & $\mathrm{M}$ & $\mathrm{H}$ & $\mathrm{L}$ & $\mathrm{L}$ \\
\hline $\begin{array}{c}\text { Finger } \\
\text { Print }\end{array}$ & $\mathrm{H}$ & $\mathrm{H}$ & $\mathrm{M}$ & $\mathrm{H}$ & M & M & $\mathrm{H}$ & $\mathrm{M}$ \\
\hline $\begin{array}{l}\text { Palm } \\
\text { Print }\end{array}$ & $\mathrm{H}$ & $\mathrm{H}$ & $\mathrm{M}$ & $\mathrm{H}$ & M & M & $\mathrm{H}$ & M \\
\hline $\begin{array}{c}\text { Hand } \\
\text { geometr } \\
y\end{array}$ & M & $\mathrm{L}$ & $\mathrm{H}$ & $\mathrm{H}$ & $\mathrm{H}$ & M & $\mathrm{M}$ & $\mathrm{M}$ \\
\hline Face & M & $\mathrm{M}$ & $\mathrm{H}$ & $\mathrm{M}$ & $\mathrm{H}$ & $\mathrm{L}$ & $\mathrm{H}$ & $\mathrm{H}$ \\
\hline Ear & $\mathrm{M}$ & $\mathrm{M}$ & $\mathrm{H}$ & $\mathrm{M}$ & M & $\mathrm{L}$ & $\mathrm{M}$ & $\mathrm{L}$ \\
\hline DNA & $\mathrm{H}$ & $\mathrm{H}$ & $\mathrm{H}$ & $\mathrm{L}$ & $\mathrm{L}$ & $\mathrm{H}$ & $\mathrm{H}$ & $\mathrm{L}$ \\
\hline Voice & $\mathrm{L}$ & $\mathrm{L}$ & $\mathrm{M}$ & $\mathrm{M}$ & $\mathrm{M}$ & $\mathrm{L}$ & $\mathrm{H}$ & $\mathrm{H}$ \\
\hline $\begin{array}{l}\text { Signatu } \\
\text { re }\end{array}$ & $\mathrm{H}$ & $\mathrm{L}$ & $\mathrm{L}$ & M & $\mathrm{H}$ & M & $\mathrm{H}$ & $\mathrm{H}$ \\
\hline $\begin{array}{l}\text { Typing } \\
\text { rhythm }\end{array}$ & $\mathrm{L}$ & $\mathrm{L}$ & $\mathrm{L}$ & $\mathrm{L}$ & M & $\mathrm{L}$ & $\mathrm{L}$ & M \\
\hline
\end{tabular}


Generally, the iris recognition systems consist of the following steps: (i) image acquisition, (ii) iris segmentation, (iii) normalization, (iv) feature extraction and (v) classification. In this paper, segmentation is achieved using Hough transform for localizing the iris and pupil regions. The segmented iris region is normalized to a rectangular block with fixed polar dimensions using Daugman's rubber sheet model. The technique includes CNN and SVM used for feature extraction and classification respectively to increase the performance of the iris recognition.

\section{METHODOLOGY}

In this section, the methodology for iris recognition is discussed. Fig. 2 shows the process diagram that has been used in this study.

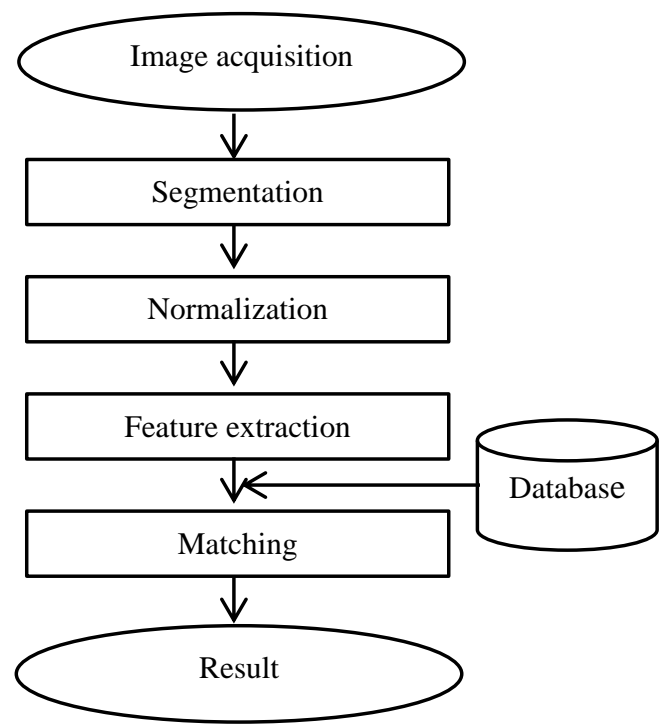

Fig 2: Flow diagram of the system employed in this study.

\subsection{Image Acquisition}

The first step of iris recognition is image acquisition deals with capturing sequence of high quality iris images from the subject using cameras and sensors. These images should clearly show the entire eye especially iris and pupil part [5], and then some preprocessing operation may be applied to enhance the quality of image e.g. Obtain images with sufficient resolution and sharpness. In this paper, CASIA-Iris V4 database is used instead of capturing eye images. CASIAIrisV4 is an extension of CASIA-IrisV3 and contains six subsets. The three subsets from CASIA-IrisV3 are CASIAIris-Interval, CASIA-Iris-Lamp, and CASIA-Iris-Twins respectively. The three new subsets are CASIA-Iris-Distance, CASIA-Iris-Thousand, and CASIA-Iris-Syn. CASIA-IrisV4 contains a total of 54,601 iris images from more than 1,800 genuine subjects and 1,000 virtual subjects. All iris images are 8 bit gray-level JPEG files, collected under near infrared illumination or synthesized [6].

\subsection{Iris Segmentation}

The next step of iris recognition is iris segmentation, is a process to isolate the actual iris region in a digital eye image. The iris region, shown in Fig. 1, can be approximated by two circles, one for the iris/sclera boundary and another, interior to the first, for the iris/pupil boundary. Hough Transformation is used to locate the circular iris region.

\subsubsection{Hough Transformation}

It is an algorithm used to compute the parameters of the geometric objects (lines and circles) in an image. The circular
Hough transform can be used to find the center coordinates and radius of the iris and pupil regions. This technique is generally used to find shapes of the objects by a voting procedure within the classes available. In the segmentation algorithm, an edge map is created by computing the gradients (first derivatives of intensity values) in an eye image. For each edge pixel in the edge map, the surrounding points on the circle at different radii are taken and votes are cast for finding the maximum values that constitute the parameters of circles in the Hough space [7]. The center coordinates and the radius are computed using the following equation:

$x_{c}^{2}+y_{c}^{2}-r^{2}=0$

The maximum point corresponds to the radius ' $r$ '; the center coordinates $\left(x_{c}, y_{c}\right)$ of the circle are given by the edge points in the Hough space.

To perform the edge detection, derivatives (gradients) are taken in the vertical direction for detecting the iris-sclera boundary, in order to reduce the influence of the eyelids that are horizontally aligned [8]. Taking only the vertical gradients for locating the iris boundary will reduce influence of the eyelids when performing circular Hough transform, and not all of the edge pixels defining the circle are required for successful localization. Not only does this make circle localization more accurate, it also makes it more efficient, since there are less edge points to cast votes in the Hough space [8]. Fig. 3 shows the pictorial representation of the Hough transformation.

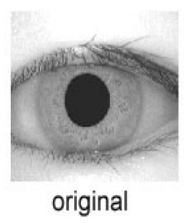

Fig 3: Segmentation
2.3 Normalization

After successfully segmented the iris region from an eye image, the circular iris region is transformed into a fixed size rectangular block. The iris regions, which have the same constant dimensions is produced in the normalization process, so that two photographs of the same iris under different conditions will have characteristic features at the same spatial location [9]. Daugman's rubber-sheet model is used here to normalize iris image.

\subsubsection{Daugman's rubber-sheet model}

The most widely used method for iris normalization is Daugman's rubber-sheet model [9], which converts the iris to a rectangular block over a doubly dimensionless nonconcentric polar coordinate system. The Daugman's rubber sheet model finds for every pixel in the iris, an equivalent position on the polar axes $(r, \theta)$ where $r$ is the radial distance and $\theta$ is the rotated angle at the corresponding radius. The radial resolution is described as the number of data points in the radial direction while the angular resolution is the number of radial lines generated around the iris region. Using equation 2 ,

$$
I[x(r, \theta), y(r, \theta)] \rightarrow I(r, \theta)
$$

The iris region is transformed to a $2 \mathrm{D}$ array with horizontal dimensions corresponding to the angular resolution and the vertical dimension to radial resolution shown in Fig. 4. 


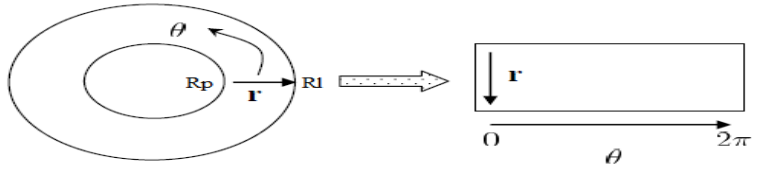

Fig 4: Daugman's Rubber-sheet Model.

Where I $(x, y)$ corresponds to the iris region, $(x, y)$ and $(r, \theta)$ are the Cartesian and normalized polar coordinates, respectively. $\theta$ ranges from 0 to $2 \pi$ and $r$ from $R p$ to $R 1$. $x(r, \theta)$ and $y(r, \theta)$ are defined as linear combinations of pupil boundary points [10].

\subsection{Feature Extraction}

The most important step in iris recognition is feature extraction. Feature extraction is a process of finding the most discriminating information present in an iris pattern. The recognition rate and run time of matching of two iris templates mostly depends on feature extraction technique. In this paper, CNN is used for extracting feature of the normalized iris image.

\subsubsection{Convolutional Neural Network}

A specific category of neural networks methods is CNN that has not only been able to learn image feature representations automatically, but they have also outperformed many conventional hand-crafted feature techniques [11]. Neural networks models have a hierarchical representation of data and depend on the computation of layers. NN models have a sequential implementation, which means, the previous layers output will be the next layers input. Every layer gives one representation level and there are a set of weights that parameterized the layers. It is also noted that, the input units linking to output units through the weights in addition to a group of biases [12]. In CNN, the weights are shared locally, which means that each location of the input has the similar weights. The filter form by the weights linked to the similar output [13].

A CNN encompasses alternating layers of locally connected convolutional layers. Every layer has the same number of filters. Down-sampling layers and the fully connected layers work as a classifier [14]. CNN architecture shown in fig 5, is effective because of having three concepts: local receptive fields, weights sharing, and down-sampling operations [15]. The local receptive field means every neuron accepts input from a small portion of the preceding layer. It has also the same size of the convolution filter. Local receptive fields are used in convolutional and down-sampling layers. The weight sharing is applied to the convolutional layer to control the capacity and to decrease the complexity of the model. Finally, the nonlinear down-sampling which used in the downsampling layers to decrease the spatial size of the image as well as decrease the number of the free parameters of the model. With these concepts, CNN is very strong and effective in learning. Moreover the details of the Convolutional Neural Networks layers are:

The Convolutional Layer: the weights in this layer are made of a set of learnable filters produced randomly and learned through the back-propagation algorithm. The feature map is the outcome of every filter that convolved through the entire image. Also, the feature maps have the same number of the applied filters in that layer [15].

As shown in Fig. 5, the first convolutional layer containing 6 filters that produced 6 feature maps which arranged together. Every feature map represents specific features from the image, for example, represented points, or represented vertical edges [16]. The convolution operation can be described as follows,

$$
x_{i}^{l}=f\left(\sum_{i \in M_{j}} x_{i}^{l-1} * k_{i j}^{l}+b_{j}^{l}\right)
$$

Here $\mathrm{j}$ is the specific convolution feature map, $\mathrm{M}_{\mathrm{j}}$ is a selection of input maps, $k_{i j}$ is the filter, $b_{j}$ is the feature map bias, 1 is the layer in the $\mathrm{CNN}$, and $\mathrm{f}$ is the activation function [14]. The ReLU is the common activation function which used to add non-linearity to the network [15].

The Pooling Layer: It implements a down-sampling operation to decrease the spatial size of the convolutional layers. First, the size of pooling mask and pooling operation type must be determined and after that applied at the pooling layer [17].

The pooling operation implemented on the pixel values captured by the pooling mask, multiply it by a trainable coefficient, after that added to a trainable bias [14]. The pooling operation can be described as follows,

$$
x_{j}^{l}=f\left(B_{j}^{l} \operatorname{pool}\left(x_{j}^{l-i}\right)+b_{j}^{l}\right)
$$

Where $x_{j}^{l}$ is the result of the down-sampling operation applied on the $\mathrm{j}^{\text {th }}$ region in the input, $x_{j}^{l-i}$ is the $\mathrm{j}^{\text {th }}$ region of interest captured by the pooling mask in the previous layer, pool is the specific operation done on the region (max or average), $B_{j}^{l}$ is a trainable coefficient, $b_{j}^{l}$ is a trainable bias,and $\mathrm{f}$ is an activation function [18]. The max pooling is the most common pooling operation and it is used in this work.

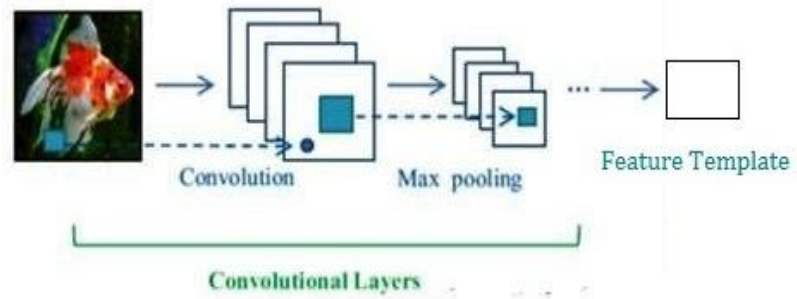

Fig 5: An illustration of the CNN architecture.

\subsection{Classification}

The template generated in the feature extraction stage needs a matching metric to measure the similarity between two iris templates. This metric gives one range of values when templates generated from the same eye are compared and another range of values when templates generated from different person's eye are compared; so that we can decide as to whether the two templates belong to the same or different persons.

\subsubsection{Support Vector Machine}

Pattern recognition is performed by Support vector machine (SVM) that works on the basis of the principle of structural risk minimization. SVM is treated as binary classifier that separates the two classes of data optimally. The two major aspects of developing SVM as a classifier is: (i) to determine the optimal hyperplane in between two separate classes of data and (ii) to transform the non-linearly separable classification problem into linearly separable problem [20,21]. Linearly separable classification problem is shown in Fig. 6 as for an example. 


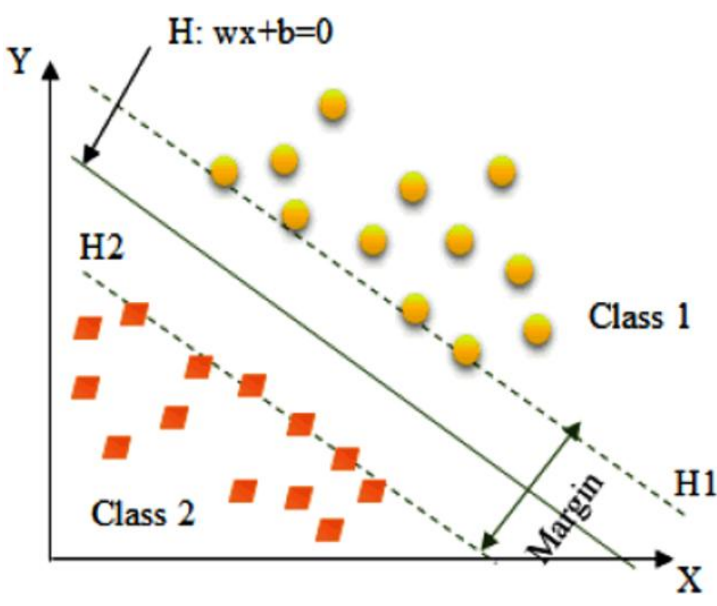

Fig 6: SVM with Linear separable data.

Let $\mathrm{x}$ is a set of input feature vector and $\mathrm{y}$ is the class label. The input feature vectors and the class label can be represented as $\left\{x_{i}, y_{i}\right\}$, where $\mathrm{i}=1,2 \ldots \mathrm{N}$ and $\mathrm{y}= \pm 1$. The separating hyperplane can be represented as follows,

$w \cdot x+b=0$

This implies,

$y_{i}\left(w \cdot x_{i}+b\right) \geq 1 ; \quad i=1,2,3 \ldots N$

$\{w, b\}$ can have numerous possible values which create separating hyperplane. It is believed that points often lie between two data classes in such a way that there is always some margin in between them. SVM maximizes this margin by considering it as a quadratic problem [22]. The SVM is used to make two possible decisions during iris recognition; acceptance or rejection of a person.

\section{RESULTS AND DISCUSSION}

The experimental results of the proposed technique on CASIA iris database are reported. 100 iris images of 100 individuals were enrolled in our system database for evaluating the performance and Intel core-i5 3.30GH processor, 4GB RAM, windows-7 operating system and matlab2019a tools were used as experimental platform. $\mathrm{CNN}$ is used to select the feature vector of iris images and got $96.3 \%$ accuracy of our proposed technique. Table 2 shows the experimental results.

A technique proposed by SG Firake et al. to compare in which Hough transform for segmentation, Daugman's rubber sheet model for normalization and Hamming distance were used for classification. Their average accuracy was $92.85 \%$ for Gabor filter [10]. A technique proposed by MH Hamd et al. to compare two feature extraction methods, PCA and Fourier descriptors (FD), in which circular Hough transform for segmentation, Daugman's rubber sheet model for normalization and Manhattan classifier for classification were used. Their average accuracy for PCA was 94\%[23]. A technique proposed by HK Rana et al. that combined PCA and DWT for extraction, in which circular Hough transform for segmentation, Daugman's rubber sheet model for normalization and SVM for classification were used. They gain the accuracy of $95.4 \%$ [20].

Table 2: Accuracy comparison of our proposed technique with others.

\begin{tabular}{|c|c|c|c|}
\hline Sl. & Authors & Methods & Accuracy \\
\hline 1. & SG Firake et al. & Gabor filter & $92.85 \%$ \\
\hline
\end{tabular}

\begin{tabular}{|c|c|c|c|}
\hline 2. & MH Hamd et al. & PCA & $94 \%$ \\
\hline 3. & HK Rana et al. & PCA + DWT & $95.4 \%$ \\
\hline 4. & MS Azam et al. & Our proposed & $96.3 \%$ \\
\hline
\end{tabular}

\section{CONCLUSIONS}

Iris recognition is a promising field of security concern that uses human iris to identify. Each individual in a population is possible to identify by calculating the iris feature. The reason why iris recognition is an attractive field is due to the fact that iris feature cannot be forgotten or lost, they are difficult to copy, share and distribute and they require the person to be present at the time of authentication. However, the enhancement of accuracy mostly depends on feature extraction and classification techniques. So that feature extraction and classification are emphasized in this study. CNN and SVM are relatively new and good performing machine learning techniques for feature extraction and classification. We have choose these technique and our experimental results have demonstrated that the proposed technique achieved good performance in accuracy. This confirms that the proposed strategy of feature extraction and classification is suitable for increasing accuracy of iris recognition.

\section{ACKNOWLEDGMENTS}

We are very much grateful to Pabna University of Science and Technology (PUST), Bangladesh, for some financial support in this study.

\section{REFERENCES}

[1] SaiyedUmer, Bibhas Chandra Dhara, BhabatoshChanda, "A Novel Cance-lable Iris Recognition System Based on Feature Learning Techniques", Elsevier Information Sciences, vol. 406-407, pp. 102-118, 2017.

[2] Imran Naseema, AffanAleemb, RobertoTogneric and Mohammed Bennamoun, "Iris recognition using classspecific dictionaries", Elsevier Computers and Electrical Engineering, vol. 62, pp. 178-193, 2016.

[3] Chiara Galdia, Michele Nappib and Jean-Luc Dugelaya, "Multimodal authentication on smartphones: Combining iris and sensor recognition for a double check of user identity", Elsevier Pattern Recognition Letters, vol. 82, pp. 144-153, 2016.

[4] HimanshuSrivastava, "A Comparison Based Study on Biometrics for Human Recognition”, IOSR Journal of Computer Engineering, vol. 15, no. 1, 2013.

[5] Rana HK, Azam MS, Akhtar MR, "Iris recognition system using PCA based on DWT", SM Journal of Biometrics \& Biostatistics vol. 2, no. 3, pp.: 1015, DOI 10.5281/zenodo.2580202, 2017

[6] Tieniu Tan ZS, Center for biometrics and security research. Available at http:// www.cbsr.ia.ac.cn/ china/ Iris\%20Databases\%20CH.asp, 2015 (accessed on 6 May 2020).

[7] PrateekVerma, MaheedharDubey, SomakBasu, Praveen Verma, "Hough Transform Method for Iris RecognitionA Biometric Approach", International Journal of Engineering and Innovative Technology (IJEIT), Volume 1, Issue 6, June 2012.

[8] PrateekVerma, MaheedharDubey, Praveen Verma, 
SomakBasu, "daughman"s algorithm method for iris Recognition-a biometric approach", International Journal of Emerging Technology and Advanced Engineering Website: www.ijetae.com (ISSN 2250-2459, Volume 2, Issue 6, June 2012.

[9] John G. Daugman. "High condence visual recognition of persons by a test of statistical independence". Pattern Analysis and Machine Intelligence, IEEE Transactions on, 15(11):1148-1161, Nov. 1993.

[10] Hamd, M. H. and Ahmed, S. K., "Biometric system design for iris recognition using intelligent algorithms", International Journal of Modern Education and Computer Science, vol. 10, no. 3, pp. 9-16,2018.

[11] O. Oyedotun and A. Khashman, "Iris nevus diagnosis: convolutional neural network and deep belief network," Turkish Journal of Electrical Engineering \& Computer Sciences, vol. 25, pp. 1106-1115, 2017.

[12] D. Scherer, A. Müller, and S. Behnke, "Evaluation of pooling operations in convolutional architectures for object recognition," Artificial Neural Networks-ICANN 2010, pp. 92-101, 2010.

[13] A. S. Al-Waisy, R. Qahwaji, S. Ipson, S. Al-Fahdawi, and T. A. Nagem, "A multi-biometric irisrecognition system based on a deep learning approach," Pattern Analysis and Applications, pp. 1-20, 2017.

[14] C. L. Lam and M. Eizenman, "Convolutional neural networks for eye detection in remote gaze estimation systems," Proceedings of the International Multi Conference of Engineers and Computer Scientists. Vol. 1. Citeseer, 2008.

[15] L. Ma, T. Tan, Y. Wang and D. Zhang, "Efficient iris recognition by characterizing key local variation", IEEE Trans on Image Process, vol. 13, no. 6, pp. 739-750, IEEE, 2004.

[16] DeepthiRampally, "ris Recognition Based on Feature
Extraction", Department of Electrical and Computer Engineering, Kansas State University, 2010.

[17] K. Nguyen, C. Fookes, A. Ross, and S. Sridharan, "Iris Recognition with Off-the-Shelf CNN Features: A Deep Learning Perspective," IEEE Access, 2017.

[18] A. S. Al-Waisy, R. Qahwaji, S. Ipson, S. Al-Fahdawi, and T. A.Nagem, "A multi-biometric iris recognition system based on a deep learning approach," Pattern Analysis and Applications, pp. 1-20, 2017.

[19] J. Nagi, F. Ducatelle, G. A. Di Caro, D. Cireşan, U. Meier, A. Giusti, F. Nagi, J. Schmidhuber, and L.M. Gambardella, "Max-pooling convolutional neural networks for vision-based hand gesture recognition," in Signal and Image Processing Applications (ICSIPA), 2011 IEEE International Conference on, 2011, pp. 342347.

[20] Rana HK, Azam MS, Akhtar MR, Quinn JMW, Moni MA, "A fast iris recognition system through optimumfeature extraction", PeerJ Computer Science, 5:e184 https://doi.org/10.7717/peerj-cs.184, 2019.

[21] Johora, FatemaTuj, Mehdi Hassan Jony, MdShakhawatHossain, and HumayanKabir. "lung cancer detection using marker-controlled watershed with svm", GUB Journal of Science and Engineering, vol. 5, no. 1, pp. 24-30, 2018.

[22] Jony, Mehdi Hassan, et al. "Detection of Lung Cancer from CT Scan Images using GLCM and SVM." 2019 1st International Conference on Advances in Science, Engineering and Robotics Technology (ICASERT). IEEE, 2019

[23] Firake SG, Mahajan PM, "Comparison of iris recognition using gabor wavelet,principal component analysis and independent component analysis", International Journal of Innovative Research in Computer and Communication Engineering, vol. 4, no. 6, pp. 12334-12342, DOI 10.15680/IJIRCCE.2016.0406293, 2016. 